\title{
A treatment of the Zeeman effect using Stokes formalism and its implementation in the Atmospheric Radiative Transfer Simulator (ARTS)
}

\author{
Richard Larsson ${ }^{\mathrm{a}, *}$, Stefan A. Buehler ${ }^{\mathrm{a}}$, Patrick Eriksson ${ }^{\mathrm{b}}$, Jana Mendrok ${ }^{\mathrm{a}}$ \\ a Division of Space Technology, Department of Computer Science, Electrical and Space Engineering, Luleå University of Technology, \\ Box 812, SE-98128 Kiruna, Sweden \\ ${ }^{\mathrm{b}}$ Department of Earth and Space Sciences, Chalmers University of Technology, SE-41296 Gothenburg, Sweden
}

\section{A R T I C L E I N F O}

\section{Article history:}

Received 13 February 2013

Accepted 6 September 2013

Available online 16 September 2013

Keywords:

Zeeman effect

Atmospheric radiative transfer

Polarization

Stokes formalism

\begin{abstract}
A B S T R A C T
This paper presents the practical theory that was used to implement the Zeeman effect using Stokes formalism in the Atmospheric Radiative Transfer Simulator (ARTS). ARTS now treats the Zeeman effect in a general manner for several gas species for all polarizations and takes into account variations in both magnetic and atmospheric fields along a full 3D geometry. We present how Zeeman splitting affects polarization in radiative transfer simulations and find that the effect may be large in Earth settings for polarized receivers in limb observing geometry. We find that not taking a spatially varying magnetic field into account can result in absolute errors in the measurement vector of at least $10 \mathrm{~K}$ in Earth magnetic field settings. The paper also presents qualitative tests for $\mathrm{O}_{2}$ lines against previous models $(61.15 \mathrm{GHz}$ line) and satellite data from Odin-SMR (487.25 GHz line), and the overall consistency between previous models, satellite data, and the new ARTS Zeeman module seems encouraging.
\end{abstract}

(c) 2013 Elsevier Ltd. All rights reserved.

\section{Introduction}

The Zeeman effect [1] occurs when an external magnetic field interacts with a molecule or an atom of total electron spin different from zero. Such an interaction will split an original energy level into several sub-levels [2]. One molecule in the atmosphere of Earth with total electron spin different from zero is $\mathrm{O}_{2}$ for which the Zeeman effect plays a crucial role in radiative transfer in

\footnotetext{
* Corresponding author. Tel.: +46 722225516 .

E-mail addresses: ric.larsson@gmail.com (R. Larsson), sbuehler@ltu.se (S.A. Buehler), patrick.eriksson@chalmers.se

(P. Eriksson), jana.mendrok@ltu.se (J. Mendrok).

URLS: http://www.sat.ltu.se (S.A. Buehler),

http://www.chalmers.se/rss (P. Eriksson),

http://www.sat.ltu.se (J. Mendrok).
}

the microwave region at lower pressures [3]. Simplistically, the Zeeman effect can be regarded as several lines shifted from a central frequency with lines polarized in a quasisymmetric manner around that central frequency. When line broadening parameters are much larger than the frequency shift, Zeeman affected radiative transfer may be physically indistinguishable from non-Zeeman affected radiative transfer. For an early review on the quantum physics of the problem see Hill [4], for a more recent review see Schadee [5] and for a detailed textbook discussion see, e.g., Berestetskii et al. [6] in the Landau and Lifshitz series on theoretical physics. For instructions on determining important parameters for the Zeeman effect see, e.g., Veseth [7] and Christensen and Veseth [8].

There are (at least) two different approaches for polarized radiative transfer simulations. We will call these the coherency formalism and the Stokes formalism, and we 
refer to Appendix A for a short description of these. Lenoir $[2,3]$ was the first to describe the Zeeman effect on radiative transfer for $\mathrm{O}_{2}$ microwave lines using coherency formalism for the atmosphere of Earth. Lenoir's approach has been incorporated into models by Rosenkranz and Staelin [9], Hufford and Liebe [10], Pardo et al. [11], von Engeln [12], Schwartz et al. [13], and Han et al. [14], usually to make temperature retrieval for specific instruments possible. Except for von Engeln [12], all of these authors seem to consider the magnetic field as constant in strength and direction throughout the propagation path (a magnetic vector rather than the full magnetic field).

There are data available from instruments with sufficient microwave spectral resolution to observe the Zeeman effect in the atmosphere of Earth for various $\mathrm{O}_{2}$ lines. Such data have been gathered by, e.g., MLS [13], Odin-SMR [15], and MAS [16,12].

The solar physics community does Zeeman affected radiative transfer using Stokes formalism. Such works include Rees et al. [17] and Berdyugina and Solanki [18]. The solar physics community uses the Zeeman effect to measure the magnetic field of stars. These measurements are usually done at shorter wavelengths and therefore for a different set of molecules and atoms than those usually considered in radiative transfer in planetary atmospheric sciences.

The Atmospheric Radiative Transfer Simulator (ARTS) $[19,20]$ handles microwave radiative transfer in any atmospheric and weak magnetic field. ARTS operates using Stokes formalism, can operate in full 3D and run simulations on various planets in the solar system. Polarization in the model was previously mainly a product of the ice cloud and surface scattering modules [21,22]. All molecular absorption/emission were assumed to be scalar before the addition of the Zeeman module. Now the Zeeman module may also be used to model the polarized radiative transfer in ARTS. ARTS can be found at http://www.sat.ltu. se/arts/ and it is both free of cost and open-source.

We will in this paper describe the implementation of the Zeeman effect in ARTS. This will be done by providing the practical theory of the Zeeman effect using Stokes formalism in Section 2. We will show examples of how ARTS handles $\mathrm{O}_{2}$ lines in the mesosphere of Earth in Section 3 as well as compare our model with previous models and data. Section 4 contains a short discussion on the results of Section 3, an outlook on what we plan to do with the newly implemented Zeeman module in the future and a short conclusion.

\section{Theory}

\subsection{Introduction}

The radiative transfer equation in local thermodynamic equilibrium without scattering is (see, e.g., [23-25] or [26])

$\frac{d \vec{I}}{d s}=-\mathbf{K}(\vec{I}-\vec{B})$,

where $\vec{I}=[I, Q, U, V]^{T}$ is the Stokes measurement vector as defined by, e.g., Eriksson et al. [20], $s$ is the path, $\mathbf{K}$ is the propagation matrix and $\vec{B}$ is the source function, usually
$[B, 0,0,0]^{T}$ with $B$ as the Planck function. Note that other terms are in use in the literature to describe $\mathbf{K}$. These include, e.g., extinction matrix and absorption matrix. The propagation matrix for a single line of a single species due to attenuation without the Zeeman effect is simply $\mathbf{K}_{J^{\prime}, J^{\prime \prime}, N^{\prime}, N^{\prime \prime} i}=\alpha_{J^{\prime}, J^{\prime \prime}, N^{\prime}, N^{\prime \prime}, i} \mathbf{I}$, where $\mathbf{I}$ is the unity matrix, $\alpha_{J^{\prime}, J^{\prime \prime}, N^{\prime}, N^{\prime \prime}, i}$ is the line absorption coefficient, $J$ is the total angular momentum quantum number, $N$ is the total angular momentum number without spin, single prime denotes the upper level, double prime denotes the lower level of the line transition and $i$ is the species. The absorption coefficient for a single line of a single species can be calculated from

$\alpha_{J^{\prime}, J^{\prime \prime}, N^{\prime}, N^{\prime \prime}, i}(\nu)=n_{i} S_{J^{\prime}, J^{\prime \prime}, N^{\prime}, N^{\prime \prime}} F^{\prime}(\nu)$,

where $n_{i}$ is the molecular number density of molecule $i$ in the atmosphere, $S_{J^{\prime} J^{\prime \prime}, N^{\prime}, N^{\prime \prime}}$ is the line strength of the $J^{\prime} \rightarrow J^{\prime \prime}$ and $N^{\prime} \rightarrow N^{\prime \prime}$ transitions, $F^{\prime}$ is some line shape function and $\nu$ is the frequency.

\subsection{Introducing the Zeeman effect}

The equation for the propagation matrix for one $J^{\prime} \rightarrow J^{\prime \prime}$ and $N^{\prime} \rightarrow N^{\prime \prime}$ line using Stokes formalism in a Zeeman affected case will be

$\mathbf{K}_{J^{\prime}, J^{\prime \prime}, N^{\prime}, N^{\prime \prime}, i}(\nu)=\mathbf{K}_{A}(\nu)+\mathbf{K}_{B}(\nu)$,

where

$\mathbf{K}_{A}(\nu)=\frac{n_{i}}{2} S_{J^{\prime} J^{\prime \prime}, N^{\prime}, N^{\prime \prime}} \sum_{M, \Delta M}\left[S_{M^{\prime}, M^{\prime \prime}} F_{A}\left(\nu, \nu_{0}+\Delta \nu_{0}\right) \boldsymbol{\Phi}_{A}\right]$

is due to attenuation and

$\mathbf{K}_{B}(\nu)=n_{i} S_{J^{\prime} J^{\prime \prime}, N^{\prime}, N^{\prime \prime}} \sum_{M, \Delta M}\left[S_{M^{\prime}, M^{\prime \prime}} F_{B}\left(\nu, \nu_{0}+\Delta \nu_{0}\right) \boldsymbol{\Phi}_{B}\right]$

is due to what is called the magneto-optic effect. The terms in the propagation matrix are cumulative, such that for all species and allowed transitions

$\mathbf{K}(\nu)=\sum_{i} \sum_{J, \Delta J, N, \Delta N} \mathbf{K}_{J^{\prime}, J^{\prime \prime}, N^{\prime}, N^{\prime \prime}, i}(\nu)$.

In Eqs. (4) and (5), $S_{M^{\prime}, M^{\prime \prime}}$ is the relative line strength of the Zeeman sub-levels, $M$ is the projection of $\vec{J}$ on the magnetic field, $F_{A / B}$ are line shape functions, $\nu_{0}$ is the frequency of the non-Zeeman affected line center, $\Delta \nu_{0}$ is the frequency shift due to the Zeeman effect, and $\boldsymbol{\Phi}_{A / B}$ are the polarization rotation matrices using Stokes formalism. The sub-indices $A$ and $B$ stand for the attenuation and the magneto-optic effect respectively. The factor $\frac{1}{2}$, in Eq. (4), is by the convention that

$$
\sum_{M, \Delta M} S_{M^{\prime}, M^{\prime \prime}} \equiv 1, \quad \operatorname{det}\left(\sum_{M, \Delta M} \Phi_{A}\right) \equiv 2, \operatorname{det}\left(\sum_{M, \Delta M} \Phi_{B}\right) \equiv 0 .
$$

The frequency shift, the relative line strength, the polarization rotation matrices and the line shapes will be discussed in Sections 2.3, 2.4, 2.5 and 2.6, respectively.

There are discrete molecular energy levels associated with the combination of quantum numbers $J, N$ and $M$. A transition between two such sets of numbers emits or absorbs radiation with the difference in energy between these levels. Various nomenclature are used in the 


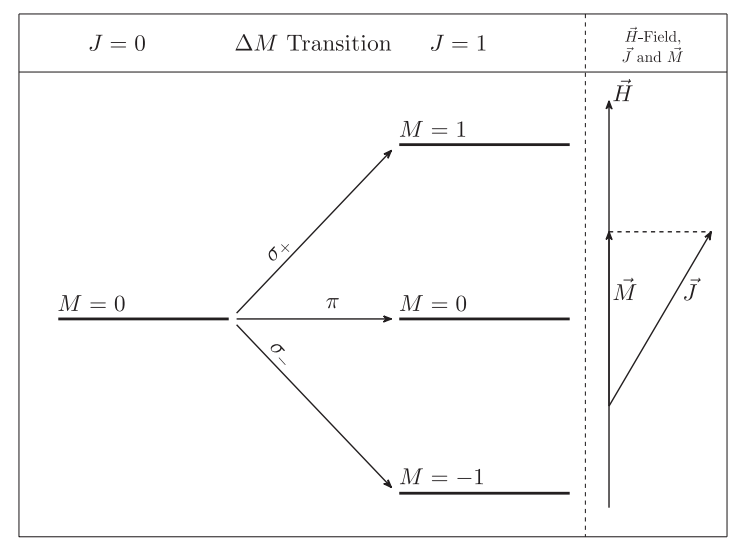

Fig. 1. An example of a Zeeman affected transition that is similar to the transition of the strong $\mathrm{O}_{2} 118.75 \mathrm{GHz}$ line. Note that $\Delta M$ is along $\vec{H}$ as shown by $M$ being a projection of $\vec{J}$ on $\vec{H}$ in the figure-the implications of this directionality and how it affects polarization is discussed in the text and is also shown in Fig. 3.

literature for the allowed types of transitions. For $\Delta J=1,0,-1$, Lenior [3] use $N^{\Delta J}$ whereas the spectroscopic community seems to use R, Q and P [5]. We will simply use $\Delta J$ when the change of $J$ demands reference. For $\Delta M=0, \pm 1$ there seems to be only one accepted notation with $\pi$ representing $\Delta M=0$ and $\sigma_{ \pm}$representing $\Delta M= \pm 1$. We will conform to this notation system. The angular momentum quantum number $J$ is limited to $|N-S| \leq J \leq N+S[6]$, where $S$ is the total electron spin quantum

The projection of the total angular momentum $\vec{J}$ on the local magnetic field vector $\vec{H}$ can take the form $M=-J, 1-J,(\cdots), J-1, J$ [3]. Transitions of the types $\Delta J=0, \pm 1$ and $\Delta M=0, \pm 1$ are the only allowed transitions, though there exists an exception for the special case of $\Delta M=\Delta J=M=0$ that is not allowed [6]. An illustration of an example transition is given in Fig. 1 showing the allowed $\Delta M$ sub-levels.

In practice, databases like, e.g., HITRAN [27,28], AFGL-86 [29] and IGRF-11 [30] can be consulted to find the parameters necessary to describe the Zeeman effect.

\subsection{Frequency shift}

Zeeman splitting refers to the shift in the upper and lower energy levels due to the external magnetic field interacting with the molecule. The energy shift of a level is

$\Delta E=-g M H \mu_{b}$,

where $H$ is the magnitude of the local magnetic field, $\mu_{b}$ is the Bohr magneton and $g$ is the Landé factor [6]. According to Lenior [3] the factor $g$ is

$g=g_{s} \frac{J(J+1)+S(S+1)-N(N+1)}{2 J(J+1)}$,

where $g_{s}$ is a particle dependent constant. Lenior [3] used $g_{s} \approx 2.00232$, the value for a free electron. It might be helpful to not use this simplification. See Table 1 for constants of $g_{s}$ that take relativistic effects and coupling between the spin and the electrons angular momentum
Table 1

Molecular $g_{s}$-factors for Eq. (9).

\begin{tabular}{llll}
\hline Molecule & $S$ & $g_{s}$-Constant & Source \\
\hline $\mathrm{O}_{2}$ & 1 & 2.002064 & Christensen and Veseth [8] \\
$\mathrm{SO}$ & 1 & 2.002106 & $\begin{array}{l}\text { Christensen and Veseth [8] } \\
\mathrm{OH}\end{array}$ \\
$\mathrm{SH}$ & $\frac{1}{2}$ & 2.00089 & Veseth [7] \\
$\mathrm{SH}$ & $\frac{1}{2}$ & 2.00089 & Veseth [7] \\
$\mathrm{NO}$ & $\frac{1}{2}$ & 2.00071 & Veseth [7] \\
$\mathrm{NS}$ & $\frac{1}{2}$ & 2.00096 & Veseth [7] \\
$\mathrm{ClO}$ & $\frac{1}{2}$ & 2.00072 & Veseth [7] \\
& & \\
\hline
\end{tabular}

Table 2

Table of relative strength, $S_{M^{\prime}, M^{\prime \prime}}$, for the allowed Zeeman sub-levels $[5,18]$.

\begin{tabular}{cll}
\hline \multicolumn{1}{c}{$\Delta J$} & $\pi$ & $\sigma_{ \pm}$ \\
\hline+1 & $\frac{3\left[(J+1)^{2}-M^{2}\right]}{2(J+1)(2 J+1)(2 J+3)}$ & $\frac{3(J+1 \pm M)(J+2 \pm M)}{4(J+1)(2 J+1)(2 J+3)}$ \\
0 & $\frac{3 M^{2}}{J(J+1)(2 J+1)}$ & $\frac{3(J \mp M)(J+1 \pm M)}{2 J(J+1)(2 J+1)}$ \\
-1 & $\frac{\left.3 J^{2}-M^{2}\right]}{2 J(2 J-1)(2 J+1)}$ & $\frac{3(J \mp M)(J-1 \mp M)}{4 J(2 J-1)(2 J+1)}$ \\
\hline
\end{tabular}

into account. We will note here that the difference in $g_{s}$ only becomes important for sensors with a very high frequency resolution (i.e. a few kilohertz for Earth conditions).

Since $J$ is $N$ for cases when $S=0$, following Eq. (8) it is clear that the Zeeman effect only occurs for molecules with $S \neq 0$. It is important to note that Eq. (8) for the level $J=0$ is reduced to $\Delta E=0$ but that there is still Zeeman splitting if the other level has $\Delta E \neq 0$.

To get the Zeeman affected frequency shift, we have to compute the difference between the change of energy in the upper and lower levels. In other words, we use

$\Delta \nu_{0}=\frac{H \mu_{0}}{h}\left(g^{\prime} M^{\prime}-g^{\prime \prime} M^{\prime \prime}\right)$

to calculate the frequency shift of each Zeeman sub-level, where $h$ is the Planck constant [6]. Prime and double prime denote the upper and lower level quantum numbers respectively.

\subsection{Relative line strength}

The relative line strength, $S_{M^{\prime}, M^{\prime \prime}}$, of the Zeeman sublevels is found in Schadee [5] but are here renormalized as in Berestetskii et al. [6]. The equations for the relative line strength depending on $\Delta J$ and $\Delta M$ can be found in Table 2. We would also like to point out that the relative line strengths of Table 2 agree with Lenior [3] for the special cases presented therein. An example of the relative frequency shift versus the relative line strength for the Zeeman sub-levels can be found in Fig. 2. 


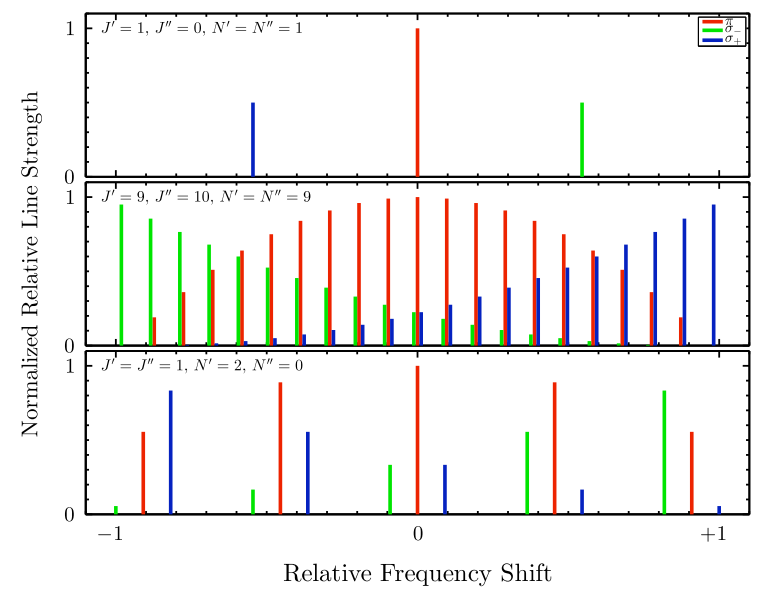

Fig. 2. Representation of normalized relative line strength versus relative frequency shift for three different $\mathrm{O}_{2}$ transitions. (These are the transitions used for radiative transfer in Figs. 4-7, in the upper panel $\nu_{0} \approx 118.75 \mathrm{GHz}$, in the middle panel $\nu_{0} \approx 61.15 \mathrm{GHz}$ line, and in the lower panel $\nu_{0} \approx 487.25 \mathrm{GHz}$.) Note that the relative line strength is normalized per transition but that frequency shift is relative to the largest split of the three examples. We see that the transition with $\Delta N \neq 0$ (lowest panel) has the largest split. We further see that the transition with $\Delta N \neq 0$ and larger $J$ (middle panel) has a larger range of frequency shifts for its sub-levels than the transition with $\Delta N \neq 0$ and lower $J$ (upper panel), but that the individual sub-levels are also farther apart for the latter.

According to Berestetskii et al. [6], $\pi$ and $\sigma_{ \pm}$each account for half of the relative line strength. For this normalization to be consistent with Eqs. (4) and (5) the $\pi$ component therefore carry a factor of 3 in Table 2, whereas the $\sigma_{ \pm}$components carry a factor of $\frac{3}{2}$ in Table 2 . That the factor is twice as large as for the $\pi$ component than for the $\sigma_{ \pm}$components can, in a simplified manner, be viewed as a geometric property.

\subsection{Polarization rotation}

The polarization induced by the Zeeman effect may be understood from the example of a local magnetic field oriented $90^{\circ}$ to the propagation path. Since $M$ is the projection of $\vec{J}$ on $\vec{H}$, it makes intuitive sense that sublevels that involve a change in the angular momentum $M$ are associated with linearly polarized radiation in the direction of $\vec{H}$, and this is indeed the case. As stated in Section 2.4, these sub-levels will account for half of the total transition strength. The other half of the power of the transition does not change the angular momentum $M$ along $\vec{H}$ and must by the same reasoning therefore be linearly polarized orthogonal to $\vec{H}$.

It is equally intuitively understandable that sub-levels with a changing $M$ will result in circular polarization in radiative transfer when $\vec{H}$ is tilted along the propagation direction vector $\vec{R}$. In the example of $\vec{R}$ fully along $\vec{H}$ the entire angular momentum change, $\Delta M$, must still be carried by the transition, which means that photons carrying a rotation vector along $\vec{R}$ will be affected. Since we observe this radiation with a sensor element directed towards $-\vec{R}$, the transition will result in circular polarization in the same right handed system as $\Delta M$. Consequently, we will observe no distinct linear polarization.

When we allow for all possible orientations of $\vec{H}$ relative to $\vec{R}$, the problem needs a more detailed mathematical treatment. If we define the angle between $\vec{H}$ and $\vec{R}$ as $\theta$ and the clockwise angle between the vertical polarization direction of the antenna and the projection of $\vec{H}$ on the plane of $\vec{R}$ as $\eta$, it has been shown by, e.g., Rees et al. [17] and Jefferies et al. [31] that

$\boldsymbol{\Phi}_{A_{\sigma_{ \pm}}}=\left[\begin{array}{cccc}1+\cos ^{2} \theta & \cos (2 \eta) \sin ^{2} \theta & \sin (2 \eta) \sin ^{2} \theta & \mp 2 \cos \theta \\ \cos (2 \eta) \sin ^{2} \theta & 1+\cos ^{2} \theta & 0 & 0 \\ \sin (2 \eta) \sin ^{2} \theta & 0 & 1+\cos ^{2} \theta & 0 \\ \mp 2 \cos \theta & 0 & 0 & 1+\cos ^{2} \theta\end{array}\right]$

and

$\boldsymbol{\Phi}_{A_{\pi}}=\left[\begin{array}{cccc}\sin ^{2} \theta & -\cos (2 \eta) \sin ^{2} \theta & -\sin (2 \eta) \sin ^{2} \theta & 0 \\ -\cos (2 \eta) \sin ^{2} \theta & \sin ^{2} \theta & 0 & 0 \\ -\sin (2 \eta) \sin ^{2} \theta & 0 & \sin ^{2} \theta & 0 \\ 0 & 0 & 0 & \sin ^{2} \theta\end{array}\right]$

describe the attenuating polarization rotation matrix of Eq. (4) that can change all of the Stokes vector components, and that

$\boldsymbol{\Phi}_{B_{\sigma_{ \pm}}}=\left[\begin{array}{cccc}0 & 0 & 0 & 0 \\ 0 & 0 & \mp 2 \cos \theta & \sin (2 \eta) \sin ^{2} \theta \\ 0 & \pm 2 \cos \theta & 0 & -\cos (2 \eta) \sin ^{2} \theta \\ 0 & -\sin (2 \eta) \sin ^{2} \theta & \cos (2 \eta) \sin ^{2} \theta & 0\end{array}\right]$

and

$\boldsymbol{\Phi}_{B_{\pi}}=\left[\begin{array}{cccc}0 & 0 & 0 & 0 \\ 0 & 0 & 0 & -\sin (2 \eta) \sin ^{2} \theta \\ 0 & 0 & 0 & \cos (2 \eta) \sin ^{2} \theta \\ 0 & \sin (2 \eta) \sin ^{2} \theta & -\cos (2 \eta) \sin ^{2} \theta & 0\end{array}\right]$

describe the magneto-optic polarization rotation matrix of Eq. (5) that can only affect the last three of the Stokes vector components. See Fig. 3 for a simple visualization of the polarization vectors and propagation direction vector $\vec{R}$.

Note that the polarization rotation matrix dependencies in

$\boldsymbol{\Phi}_{A / B}=\left[\begin{array}{cccc}A_{I} & A_{Q} & A_{U} & A_{V} \\ A_{Q} & A_{I} & B_{V} & B_{U} \\ A_{U} & -B_{V} & A_{I} & -B_{Q} \\ A_{V} & -B_{U} & B_{Q} & A_{I}\end{array}\right]$

show the contribution to the polarization rotation matrix due to the attenuation $(A)$ and the magneto-optic effect $(B)$, with the indices representing the main contributing Stokes parameter. Eqs. (11) and (12) agree with our intuitive description above, and Eqs. (13) and (14) will rotate the polarization. The latter point is important to explain why, e.g., $B_{V}$, which only affects linear polarization, is said to be from $V$, which is the circular polarization parameter of the Stokes vector. $B_{V}$ is strongest when $\vec{R}$ is along $\pm \vec{H}$, which cancels all other magneto-optic effects and implies that 


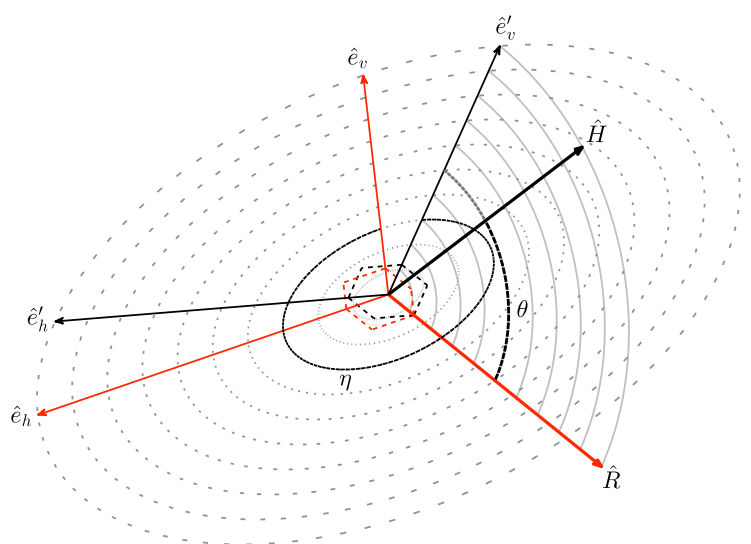

Fig. 3. Geometry of the propagation, the local magnetic field and polarization axes. All vectors in the plot are of the same length. Specifically, $\hat{e}_{v}$ is the vertical polarization axis of the sensor, $\hat{e}_{v}^{\prime}$ is the vertical polarization axis along the magnetic field $\hat{H}$ in the plane of the direction of propagation $\hat{R}, \hat{e}_{h}$ is the horizontal polarization axis of the sensor, $\hat{e}_{h}^{\prime}$ is the horizontal polarization axis perpendicular to $\hat{H}$ in the plane of $\hat{R}, \theta$ is the angle between $\hat{H}$ and $\hat{R}$, and $\eta$ is the clockwise angle between $\hat{e}_{v}^{\prime}$ and $\hat{e}_{v}$ (or between $\hat{e}_{h}^{\prime}$ and $\hat{e}_{h}$ ). Note that $\hat{e}_{v}, \hat{e}_{v}^{\prime}, \hat{e}_{h}$ and $\hat{e}_{h}^{\prime}$ are always in the plane of $\hat{R}$. Also note that $\hat{e}_{v}^{\prime}, \hat{R}$ and $\hat{H}$ are always in the plane of $\hat{e}_{h}^{\prime}$. The angles $\theta$ and $\eta$ are important for the polarization rotation matrices described by Eqs. (11)-(14).

the medium acts like a Faraday rotator by allowing one type of circular polarization to traverse the medium faster than the other, causing a phase delay that in turn rotates the linear polarization. Similar phase delays exist for linear polarization, as can be understood from $B_{Q}$ and $B_{U}$. We offer our own derivation of the polarization matrix for both the attenuation and the magneto-optic effect in Appendix A.

\subsection{Line shape}

It is conventional to define the line shape functions $F_{A / B}\left(\nu, \nu_{0}+\Delta \nu_{0}\right) \equiv F_{A / B}\left(\nu^{\prime}, a\right)$, where

$\nu^{\prime}=\frac{\nu_{0}+\Delta \nu_{0}-\nu}{\Delta \nu_{D}}$

represents the frequency difference from the line center in units of Doppler broadening width $\left(\Delta \nu_{D}\right)$ and

$a=\frac{\gamma}{4 \pi \Delta \nu_{D}}$

represents the ratio between pressure broadening width $(\gamma)$ and Doppler broadening width. From, e.g., Jefferies et al. [31] and Landi Degl'innocenti [32] we find a developed and an applied theory on the line shapes of the attenuation and the magneto-optic effect. Following their examples, the line shape of attenuation around the central frequency is the Voigt function

$F_{A}\left(\nu^{\prime}, a\right)=\frac{a}{\pi} \int_{-\infty}^{+\infty} \frac{e^{-y^{2}}}{\left(\nu^{\prime}-y\right)^{2}+a^{2}} d y$

and the line shape for the magneto-optic effect around the central frequency is the Faraday-Voigt function

$F_{B}\left(\nu^{\prime}, a\right)=\frac{1}{2 \pi} \int_{-\infty}^{+\infty} \frac{\left(\nu^{\prime}-y\right) e^{-y^{2}}}{\left(\nu^{\prime}-y\right)^{2}+a^{2}} d y$.

We find from, e.g., Sampoorna et al. [33] that we can use a renormalized Faddeeva function

$w(z)=F_{A}+i F_{B}=\frac{1}{\pi^{2}} \int_{-\infty}^{+\infty} \frac{e^{-y^{2}}}{z-y} d y$,

where $z=\nu^{\prime}+i a$ to describe both the line shape functions.

\section{Model and results}

This section will show and discuss the qualitative differences that the ARTS Zeeman module offers for radiative transfer simulations when active. The section will also show qualitative tests of how the ARTS Zeeman module compares to previous models and satellite measurements.

The atmospheres used for all the figures and comparisons herein are from AFGL 1986 [29]. The atmospheres are numerically defined up to $120 \mathrm{~km}$ - everything above is ignored and considered vacuum. The only molecule from which lines are considered in our calculations is $\mathrm{O}_{2}$. The magnetic field used is from IGRF-11 [30] and we do not take secular variations into account. We use the algorithm by Zaghloul and Ali [34] $]^{1}$ to estimate a solution to the Faddeeva function in all simulations below.

Radiative intensities presented in this section are all in Rayleigh-Jeans brightness temperature $T_{b}$. The Stokes formalism as defined by Eriksson et al. [20] implies that $I$ is the total intensity, that the horizontal linear polarization can be read from $I-Q$, vertical linear polarization can be read from $I+Q,-45^{\circ}$ linear polarization from $I-U, 45^{\circ}$ linear polarization from $I+U$, right circular polarization from $I-V$ and that the left circular polarization can be read from $I+V$.

\subsection{Properties of the model}

A simple comparison between non-/ and Zeeman affected radiative transfer for various tangent altitudes above $\left(65^{\circ} \mathrm{N}, 133^{\circ} \mathrm{E}\right)$ is shown in Fig. 4. Fig. 4 mainly demonstrates the effect of the Zeeman module in ARTS for one magnetic field, satellite position, viewing direction, atmosphere and so on, and the results are not general. We see that there is almost no difference between non-/ and Zeeman affected $I-Q$ brightness temperature $T_{b}$. Contrary to this, we also see that there is a huge difference between non-/ and Zeeman affected $I+Q$. In other words, it is clear that the Zeeman splitting affects different types of polarization in different ways. Theory states that $-45^{\circ}$ polarization and $45^{\circ}$ polarization should give roughly similar resulting $T_{b}$, and this is indeed the case. For circular polarization there is a distinguishable power skewness to the right of the central frequency for $I-V$ and to the left of the central frequency for $I+V$. The asymmetric $T_{b}$

\footnotetext{
${ }^{1}$ Reimplemented in $\mathrm{C}++$ by Steven G. Johnson under the MIT License (attainable through http://ab-initio.mit.edu/Faddeeva).
} 


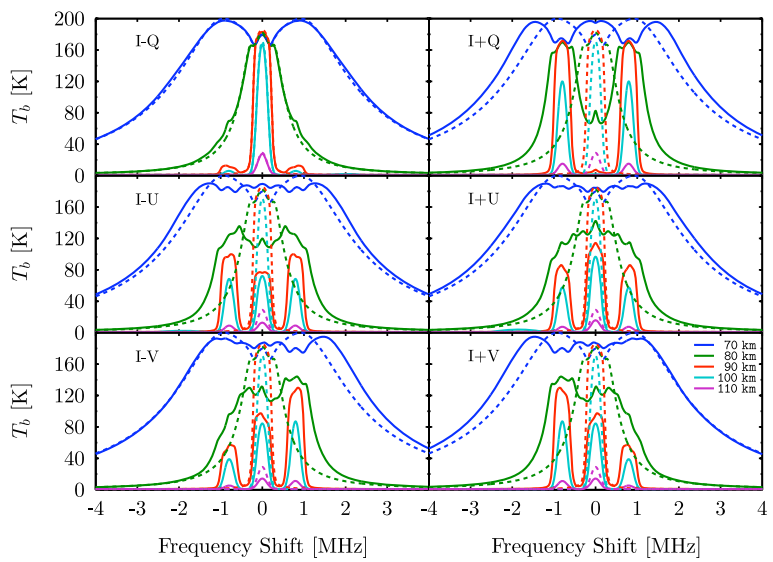

Fig. 4. Comparison between a Zeeman module and a non-Zeeman module affected line. The direction of $\vec{R}$ at the tangent point is northward and the angle between $\vec{R}$ and $\vec{H}$ is approximately $100^{\circ}$. Frequency shift is given with respect to the $\mathrm{O}_{2} 118.75 \mathrm{GHz}$ line. Dashed lines represent the case when the Zeeman effect is ignored and solid lines represent Zeeman affected radiances. This figure is discussed further in the text.

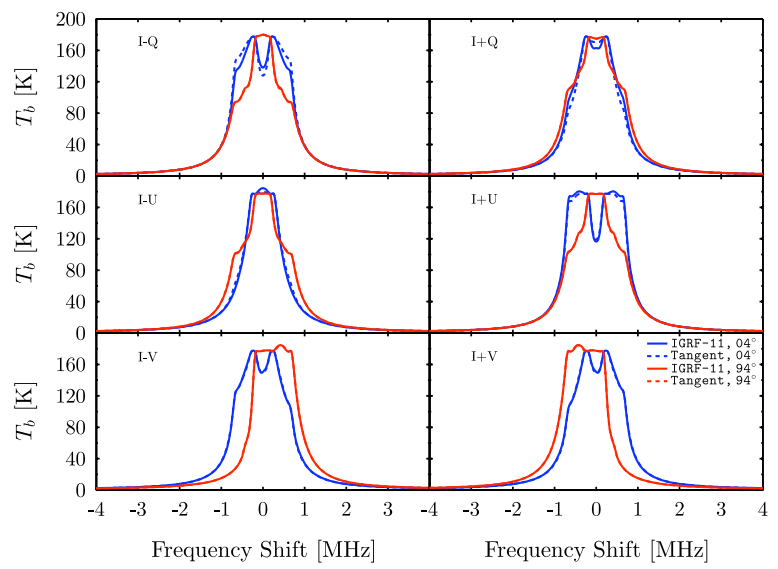

Fig. 5. Tangent point limb sounding radiative transfer model comparison between a varying local magnetic field and a constant local magnetic field $\vec{H}$ from the tangent point. Dashed lines represent the tangent point $\vec{H}$ simulations and solid lines use a full IGRF-11 field. The tangent point of this plot is at $\left(0^{\circ} \mathrm{N}, 0^{\circ} \mathrm{E}\right)$ and $80 \mathrm{~km}$. The azimuth angle of $\vec{R}$ at the tangent is given in the legends. Blue approximates $\theta$ at $90^{\circ}$ and red approximates $0^{\circ}$. The $\mathrm{O}_{2}$ line is the $118.75 \mathrm{GHz}$ line. (For interpretation of the references to color in this figure caption, the reader is referred to the web version of this paper.)

frequency skewness is to be expected from the quasisymmetry of circular polarization in $\sigma_{ \pm}$due to its mirrored frequency shift and relative line strength.

We think that it is important to note the possibility of varying the local magnetic field vector along the propagation path in ARTS and how this affects radiative transfer as compared to using a non-varying local magnetic field vector, which contrasts the ARTS Zeeman module to the most previous Zeeman capable radiative transfer models. Fig. 5 shows such a comparison for two cases when the angle between $\vec{H}$ and $\vec{R}$ is approximately $0^{\circ}$ and $90^{\circ}$ respectively when the local magnetic field vector at the tangent point is used throughout the comparative run. We see that it is preferable to define $\vec{H}$ as part of a field Failing to do so may introduce absolute errors in $T_{b}$ of at least $10 \mathrm{~K}$. During the run described above, the magnetic field in ARTS changed about $1.5 \mu \mathrm{T}$ in strength, $\theta$ changed with about $11^{\circ}$ and $\eta$ changed with about $29^{\circ}$.

\subsection{Qualitative rest results}

We have qualitatively compared our model to the simulation results in Fig. 5.6 of von Engeln [12]. The results of this comparison can be found in Fig. 6. We find that our models qualitatively agree well for all but the outermost lines at 90 and $100 \mathrm{~km}$ tangent point heights. We do not understand the reason for this discrepancy. Comparing our results with results of another model [10], Fig. 2 of Hartmann et al. [16], however, we find that the consistently concave shape is reproduced in ARTS but not by von Engeln [12]. This second comparison strengthens our belief that the implementation in ARTS is valid.

A qualitative comparison with data from Odin-SMR, see Fig. 7, shows that the implemented Zeeman module more accurately describes the breadth of the line absorption in real sub-millimeter wave limb sounding data than a similar run without the Zeeman module. This qualitative comparison makes it clear that, e.g., temperature retrieval at lower pressures using $\mathrm{O}_{2}$ lines must take the Zeeman effect into account.

\section{Summary, conclusions and outlook}

This paper has presented a practical approach to the theory of the Zeeman effect in Stokes formalism in the atmospheric sciences. Furthermore, we have presented important features of the implemented ARTS Zeeman module, we have shown that the ARTS Zeeman module

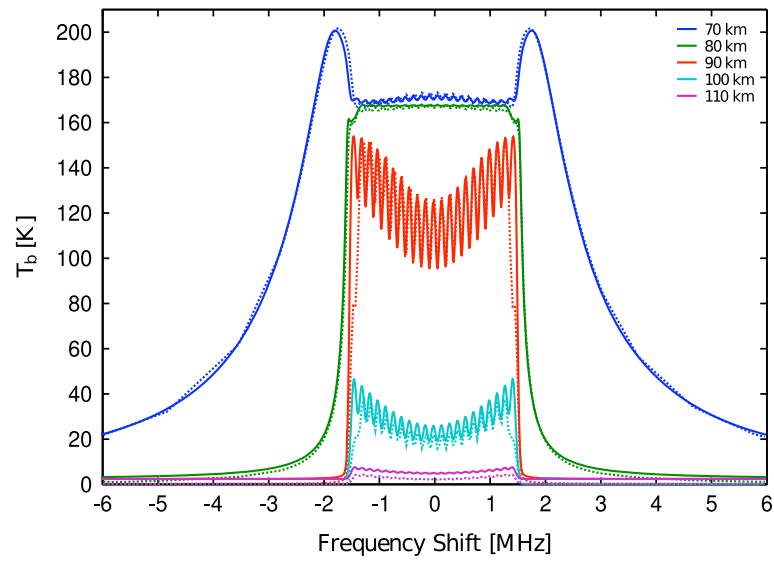

Fig. 6. Qualitative comparison between the implemented model in ARTS (solid lines) and the forward model as used by von Engeln [12] (dotted lines) in mid-latitude summer atmospheric conditions for the $N^{\prime}=N^{\prime \prime}=J^{\prime}=9$ and $J^{\prime \prime}=10$ line. Different colors indicate different tangent altitudes. There is a qualitative overlap between the models with some differences, likely due to the use of slightly different atmospheres. However, there are very clear differences at 90 and $100 \mathrm{~km}$. It seems that the two models disagree about where the outermost peaks should occur. For more about this figure see the text. (For interpretation of the references to color in this figure caption, the reader is referred to the web version of this paper.) 


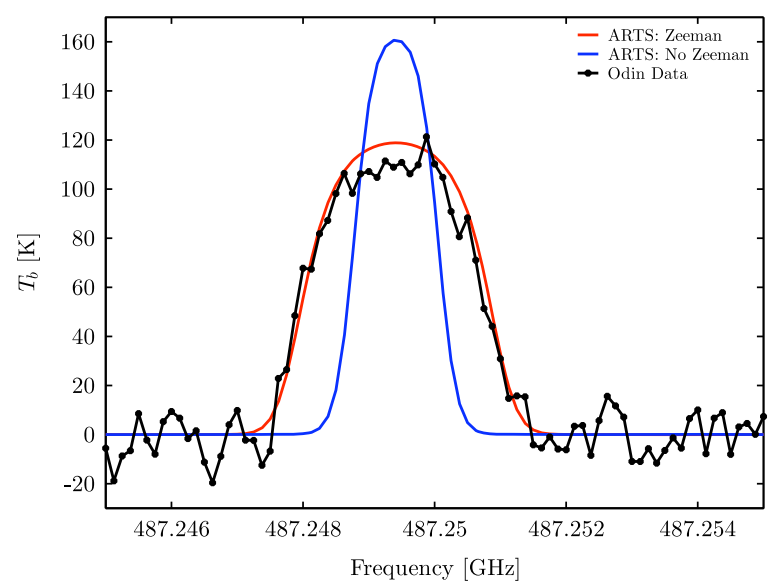

Fig. 7. Odin-SMR data collected at high frequency resolution for the $\mathrm{O}_{2}$ $487 \mathrm{GHz}$ lines show that there is a qualitative necessity to consider the Zeeman effect. This plot was selectively chosen out of a larger set to demonstrate the importance of the new module but more work is still required to retrieve the true temperature. The data was gathered with an integration time of approximately $1.8 \mathrm{~s}$, with a frequency resolution of $125 \mathrm{kHz}$ and with a $45^{\circ}$ polarized antenna. The approximative position of the satellite is at $589 \mathrm{~km}$ height, with coordinates $\left(35.57^{\circ} \mathrm{N}, 1.66^{\circ} \mathrm{W}\right)$, and the line of sight of the satellite is $111.77^{\circ}$ zenith and $-9.46^{\circ}$ azimuth hitting a tangent point at approximately $92 \mathrm{~km}$ of height.

mostly agrees with previous models, and we have shown that ARTS now represents measured satellite signals from the upper atmosphere better than before. The advantage of ARTS using Stokes parameterization is that it eases interpretation of the simulation results and makes the integration of additional modules easier. The ARTS Zeeman module seems to represent the physics of the Zeeman effect in atmospheric radiative transfer well.

As an outlook, the ARTS Zeeman module offers the opportunity to explore several interesting problems in the atmospheric sciences. The Odin-SMR data could be revisited to retrieve the corrected temperature profiles. The ARTS Zeeman module may also be used to better determine the errors associated with $\mathrm{ClO}$, $\mathrm{NO}$ and $\mathrm{OH}$ retrieval for models neglecting the Zeeman effect of these species. We are looking into combining the ARTS Zeeman module with ground-based measurements to interpret the results better. We are also investigating the possible use of the ARTS Zeeman module outside the atmosphere of Earth. For instance, both $\mathrm{O}_{2}$ and $\mathrm{NO}$ are relatively common in the atmosphere of Mars, which means that instrument parameters for future missions may be determined.

\section{Acknowledgments}

Acknowledgement is due to all those in the ARTS user community who have contributed to the ARTS development.

We would also particularly like to thank Axel von Engeln for helping us to understand his simulations.

\section{Appendix A. Translating the propagation matrix}

In coherency formalism the propagation matrix is a complex $2 \times 2$-matrix, with combinations of elements constituting a physical meaning. In Stokes formalism the propagation is a real $4 \times 4$-matrix, with each element carrying its own physical meaning. Both formalisms carry many innate symmetries, as is clear from the physics (e.g. polarization rotation), and they of course describe the same physical processes.

To our knowledge the Zeeman effect has so far only been described in terms of coherency formalism in the Earth atmospheric sciences. We therefore think it prudent to show the steps necessary to translate between the coherency formalism and the Stokes formalism for the Zeeman effect. The radiative transfer equation without scattering for local thermodynamic equilibrium using Stokes formalism can be found in Section 2.1 as Eq. (1). Equivalently, the radiative transfer equation using coherency formalism [3] is

$\frac{d \boldsymbol{S}}{d s}=-\left(\boldsymbol{G} \boldsymbol{S}+\boldsymbol{S} \boldsymbol{G}^{\dagger}\right)+\left(\boldsymbol{G}+\boldsymbol{G}^{\dagger}\right) B$

where $\boldsymbol{G}$ is the complex propagation matrix, $\boldsymbol{S}$ is the radiative coherency matrix containing information equivalent to that in the Stokes vector, and $B$ is the Plank function. Note that $\dagger$ symbolizes the complex transpose.

From Lenior [3], we find that the propagation matrix is $\boldsymbol{G}_{m}=\boldsymbol{A}_{m}+i \boldsymbol{B}_{m}$, where the subindex $m$ denotes a local magnetic field vector coordinate system throughout this section. These coherency matrices are

$$
\begin{aligned}
\boldsymbol{A}_{m} & =\left[\begin{array}{cc}
A_{1} & i A_{3} \\
-i A_{3} & A_{2}
\end{array}\right] \text { and } \\
i \boldsymbol{B}_{m} & =\left[\begin{array}{cc}
i B_{1} & B_{3} \\
-B_{3} & i B_{2}
\end{array}\right],
\end{aligned}
$$

where $\boldsymbol{A}_{m}$ is the attenuation matrix that is Hermitian $\left(\boldsymbol{A}_{m}=\boldsymbol{A}_{m}^{\dagger}\right.$ )and $i \boldsymbol{B}_{m}$ is the magneto-optic effect matrix that is anti-Hermitian $\left(\boldsymbol{i} \boldsymbol{B}_{m}=-i \boldsymbol{B}_{m}^{\dagger}\right)$. The attenuation matrices for $\pi$ and $\sigma_{ \pm}$can be found in Lenior [3]. Due to how we have defined $\boldsymbol{A}_{m}$ and $\boldsymbol{B}_{m}$ above, if the values in $\boldsymbol{A}_{m}$ and $\boldsymbol{B}_{m}$ only depend on $\theta$ (see Fig. 3) then $\boldsymbol{A}_{m}=f(\nu) \boldsymbol{B}_{m}^{*}$, where $f(\nu)$ is real $\left(\boldsymbol{A}_{m}\right.$ and $\boldsymbol{B}_{m}$ remain similar in form but their effective values follows separate functions of frequency). Note that $*$ represents the element-wise conjugate. We will treat the attenuation and the magneto-optic effect separately in the derivation below.

The transformation from coherency formalism to Stokes formalism $(\mathbf{S} \Rightarrow \vec{I})$ is

$\boldsymbol{S}=\left[\begin{array}{ll}S_{11} & S_{12} \\ S_{21} & S_{22}\end{array}\right] \Rightarrow \vec{I}=\left[\begin{array}{c}S_{11}+S_{22} \\ S_{11}-S_{22} \\ \left(S_{12}+S_{21}\right) \\ i \\ \left(S_{21}-S_{12}\right)\end{array}\right]$,

where the components are in energy and not amplitude of the wave (see, e.g., Mishchenko et al. [25] and von Engeln [12] to find these definitions). When looking in more detail at this transformation it becomes clear that

$\boldsymbol{S}=\boldsymbol{a}_{I} I+\boldsymbol{a}_{Q} Q+\boldsymbol{a}_{U} U+\boldsymbol{a}_{V} V$

where

$\boldsymbol{a}_{I}=\left[\begin{array}{ll}1 & 0 \\ 0 & 1\end{array}\right], \quad \boldsymbol{a}_{Q}=\left[\begin{array}{cc}1 & 0 \\ 0 & -1\end{array}\right]$, 
$\boldsymbol{a}_{U}=\left[\begin{array}{ll}0 & 1 \\ 1 & 0\end{array}\right], \quad \boldsymbol{a}_{V}=\left[\begin{array}{cc}0 & i \\ -i & 0\end{array}\right]$.

It is now possible to use Eqs. (A.(1) and A.2) to transform a given complex coherency propagation matrix, $\boldsymbol{G}_{m}$, into the Stokes propagation matrix, $\boldsymbol{K}_{m}$, of Eq. (1). We see that this is equivalent to stating that

$\boldsymbol{G}_{m} \boldsymbol{S}+\boldsymbol{S} \boldsymbol{G}_{m}^{\dagger} \Rightarrow \boldsymbol{K}_{m} \vec{I}$

which can be divided into two parts, the attenuation

$\boldsymbol{A}_{m} \boldsymbol{S}+\boldsymbol{S} \boldsymbol{A}_{m} \Rightarrow \boldsymbol{K}_{A, m} \vec{I}$

and the magneto-optic effect

$i\left(\boldsymbol{B}_{m} \boldsymbol{S}-\boldsymbol{S B}_{m}\right) \Rightarrow \boldsymbol{K}_{B, m} \vec{I}$,

where it is clear that $\boldsymbol{K}_{m}=\boldsymbol{K}_{A, m}+\boldsymbol{K}_{B, m}$ and we must remember that $\boldsymbol{K}_{m}$ is always real but $\boldsymbol{G}_{m}$ is not always real. It is quite tedious, but straightforward, to use Eq. (A.3) to solve the above expressions for $\boldsymbol{K}_{m}$. We get the attenuation propagation matrix

$\boldsymbol{K}_{A, m}=\left[\begin{array}{cccc}A_{1}+A_{2} & A_{1}-A_{2} & 0 & 2 A_{3} \\ A_{1}-A_{2} & A_{1}+A_{2} & 0 & 0 \\ 0 & 0 & A_{1}+A_{2} & 0 \\ 2 A_{3} & 0 & 0 & A_{1}+A_{2}\end{array}\right]$,

and the magneto-optic effect matrix

$\boldsymbol{K}_{B, m}=\left[\begin{array}{cccc}0 & 0 & 0 & 0 \\ 0 & 0 & 2 B_{3} & 0 \\ 0 & -2 B_{3} & 0 & -B_{1}+B_{2} \\ 0 & 0 & B_{1}-B_{2} & 0\end{array}\right]$.

The above expressions assume that the main axis is determined from the local magnetic field vector rather than from an external sensor. This is quite inconvenient from a modeling standpoint as it makes linear polarization difficult to track along the propagation path. It is therefore necessary to rotate Eq. (1) from the local magnetic field vector coordinate system to a sensor dependent coordinate system using

$\boldsymbol{L}^{-1} \frac{d \vec{I}}{d s}=-\boldsymbol{K}_{m} \boldsymbol{L}^{-1} \vec{I}+\boldsymbol{K}_{m} \boldsymbol{L}^{-1} \vec{B}$,

with

$\boldsymbol{L}=\left[\begin{array}{cccc}1 & 0 & 0 & 0 \\ 0 & \cos 2 \eta & -\sin 2 \eta & 0 \\ 0 & \sin 2 \eta & \cos 2 \eta & 0 \\ 0 & 0 & 0 & 1\end{array}\right]$,

where $\eta$ is the same as in Fig. 3. The above notations ensure that $\boldsymbol{K}_{m}$ acts in the magnetic coordinate system on $\vec{I}_{m}=\boldsymbol{L}^{-1} \vec{I}$, and that the result $d \vec{I}_{m} / d s=\boldsymbol{L}^{-1} d \vec{I} / d s$ also is in the magnetic coordinate system. The propagation matrix we use in ARTS to always remain in the sensor determined coordinate system is thus derived from Eq. (A.6). We get that

$\boldsymbol{K}=\boldsymbol{L} \boldsymbol{K}_{m} \boldsymbol{L}^{-1}$,

with $\boldsymbol{K}_{m}=\boldsymbol{K}_{A, m}+\boldsymbol{K}_{B, m}$ as above.

\section{References}

[1] Zeeman P. On the influence of magnetism on the nature of the light emitted by a substance. Philos Mag 1897;43:226.

[2] Lenoir WB. Propagation of partially polarized waves in a slightly anisotropic medium. J Appl Phys 1967;38(13):5283-90.

[3] Lenoir WB. Microwave spectrum of molecular oxygen in the mesosphere. J Geophys Res 1968;73(1):361-76.

[4] Hill EL. On the Zeeman effect in doublet band spectra. Phys Rev 1929;34:1507-16. http://dx.doi.org/10.1103/PhysRev.34.1507.

[5] Schadee A. On the Zeeman effect in electronic transitions of diamotic molecules. J Quant Spectrosc Radiat Transfer 1978;19: 517-31. http://dx.doi.org/10.1016/0022-4073(78)90020-1.

[6] Berestetskii VB, Lifshitz EM, Pitaevskii LP. Landau and Lifshitz course of theoretical physicsquantum electrodynamics. 2nd ed.. Pergamon Press; 1980.

[7] Veseth L. Relativistic corrections to the Zeeman effect in diatomic molecules. J Mol Struct 1977;66(2):259-71. http://dx.doi.org/ 10.1016/0022-2852(77)90216-8.

[8] Christensen $\mathrm{H}$, Veseth L. On the high-precision Zeeman effect in $\mathrm{O}_{2}$ and SO. J Mol Struct 1978;72(3):438-44. http://dx.doi.org/10.1016 0022-2852(78)90142-X.

[9] Rosenkranz PW, Staelin DH. Polarized thermal microwave emission from oxygen in the mesosphere. Radio Sci 1988;23(5):721-9.

[10] Hufford GA, Liebe HJ. Millimeter-wave propagation in the mesosphere. Technical Report, NTIA-Report; 1989.

[11] Pardo JR, Pagani L, Gerin M, Prigent C. Evidence of the Zeeman splitting in the $2_{1} \rightarrow 0_{1}$ rotational transition of the atmospheric ${ }^{16} \mathrm{O}^{18} \mathrm{O}$ molecule from ground-based measurements. J Quant Spectrosc Radiat Transfer 1995;54(6):931-43.

[12] von Engeln A. Satellite based temperature profile determination using passive microwave and radio occultation instruments. Berichte aus dem Institut fuer Umweltphysik, Logos Verlag Berlin. PhD thesis, University of Bremen; 2000. ISBN: 3-89722-453-4.

[13] Schwartz MJ, Read WG, Van Snyder W. EOS MLS forward model polarized radiative transfer for Zeeman-split Oxygen lines. IEEE Trans Geosci Remote 2006;44(5):1182-91. http://dx.doi.org/10.1109/ TGRS.2005.862267.

[14] Han Y, van Delst P, Weng F. An improved fast radiative transfer model for special sensor microwave imager/sounder upper atmosphere sounding channels. J Geophys Res 2010;115:D15109. http://dx.doi.org/10.1029/ 2010JD013878.

[15] Murtagh D, Frisk U, Merino F, Ridal M, Jonsson A, Stegman J, et al. An overview of the odin atmospheric mission. Can J Phys 2002;80 (4):309-19. http://dx.doi.org/10.1139/P01-157.

[16] Hartmann GK, Degenhardt W, Richards ML, Liebe HJ, Hufford GA, Cotton MG, et al. Zeeman splitting of the 61 gigahertz oxygen $\left(\mathrm{O}_{2}\right)$ line in the mesosphere. Geophys Res Lett 1996;23(17):2329-32.

[17] Rees DE, Murphy GA, Durrant CJ. Stokes profile analysis and vector magnetic fields, II. Formal numerical solutions of the stokes transfer equations. Astrophys J 1989;339:1093-106. http://dx.doi.org/ 10.1086/167364.

[18] Berdyugina SV, Solanki SK. The molecular Zeeman effect and diagnostics of solar and stellar magnetic fields-I. Theoretical spectral patterns in the Zeeman regime. Astron Astrophys 2002: 385. http://dx.doi.org/10.1051/0004-6361:20020130.

[19] Buehler SA, Eriksson P, Kuhn T, von Engeln A, Verdes C. ARTS the atmospheric radiative transfer simulator. J Quant Spectrosc Radiat Transfer 2005;91(1):65-93. http://dx.doi.org/10.1016/j.jqsrt. 2004.05.051.

[20] Eriksson P, Buehler SA, Davis CP, Emde C, Lemke O. ARTS, the atmospheric radiative transfer simulator, version 2. J Quant Spectrosc Radiat Transfer 2011;112(10):1551-8. http://dx.doi.org/ 10.1016/j.jqsrt.2011.03.001.

[21] Emde C, Buehler SA, Davis C, Eriksson P, Sreerekha TR, Teichmann CJ. A polarized discrete ordinate scattering model for simulations of limb and nadir longwave measurements in 1D/3D spherical atmospheres. J Geophys Res 2004;109(D24):D24207. http://dx.doi.org/ 10.1029/2004JD005140.

[22] Davis C, Emde C, Harwood R. A 3D polarized reversed Monte Carlo radiative transfer model for $\mathrm{mm}$ and sub-mm passive remote sensing in cloudy atmospheres. IEEE Trans Geosci Remote 2005;43 (5):1096-101. http://dx.doi.org/10.1109/TGRS.2004.837505.

[23] Emde C. A polarized discrete ordinate scattering model for radiative transfer simulations in spherical atmospheres with thermal source. PhD thesis, University of Bremen; 2005. ISBN: 3-8325-0885-4.

[24] Mishchenko MI, Hovenier JW, Travis LD, editors. Light scattering by nonspherical particles. Academic Press; 2000 ISBN: 0-12-498660-9. 
[25] Mishchenko MI, Travis LD, Lacis AA. Scattering, absorption, and emission of light by small particles. Cambridge University Press; 2002 ISBN: 0-521-78252-X.

[26] del Toro Iniesta JC. Introduction to spectropolarimetry. Cambridge University Press; 2004.

[27] Rothman LS, Jacquemart D, Barbe A, Benner DC, Birk M, Brown LR, et al. The HITRAN 2004 molecular spectroscopic database. J Quant Spectrosc Radiat Transfer 2005;96:139-204. http://dx.doi.org/ 10.1016/j.jqsrt.2004.10.008.

[28] Rothman LS, Gordon IE, Barbe A, Benner DC, Bernath PF, BirK M, et al. The HITRAN 2008 molecular spectroscopic database. J Quant Spectrosc Radiat Transfer 2009;110:533-72. http://dx.doi.org/ 10.1016/j.jqsrt.2009.02.013.

[29] Anderson GP, Clough SA, Kneizys FX, Chetwynd JH, Shettle EP. AFGL atmospheric constituent profiles $(0-120 \mathrm{~km})$. Technical Report, AFGL; 1986. TR-86-0110.
[30] Finlay CC, Maus S, Beggan CD, Bondar TN, Chambodut A, Chernova TA, et al. International geomagnetic reference field: the eleventh generation. Geophys J Int 2010;183:1216-30.

[31] Jefferies J, Lites BW, Skumanich A. Transfer of line radiation in a magnetic field. Astrophys J 1989;343:920-35.

[32] Landi Degl'innocenti E. MALIP-a programme to calculate the Stokes parameters profiles of magnetoactive Frauenhofer lines. Astron Astrophys Suppl Ser 1976;25:379-90.

[33] Sampoorna M, Nagendra KN, Frisch H. Generalized Voigt functions and their derivatives. J Quant Spectrosc Radiat Transfer 2007;104: 71-85.

[34] Zaghloul MR, Ali AN. Algorithm 916: computing the Faddeyeva and Voigt functions. Trans Math Software 2011;38(2). 